KAIST-TH 2000/13

hep-ph/0011275

\title{
Bounds on the Mass and Coupling Constant of Radion in the Randall-Sundrum Theory
}

\author{
Saebyok Bae ${ }^{*}$ and Hong Seok Lee ${ }^{\dagger}$ \\ Department of Physics, Korea Advanced Institute of Science and Technology \\ Taejon 305-701, Korea
}

\begin{abstract}
Assuming the Goldberger-Wise mechanism, we investigated the effective potential at the one-loop level in the Randall-Sundrum theory. We found the lower and upper bounds of the radion mass $m_{\phi}$ and the radion-SM coupling constant, $0.8 \mathrm{GeV} \lesssim m_{\phi} \lesssim 260 \mathrm{GeV}$ and $1400 \mathrm{GeV} \lesssim \Lambda_{\phi} \lesssim 1500 \mathrm{GeV}$ for the one-loop level potential. These bounds were determined from two constraints of warp factor $=O\left(M_{W} / M_{\mathrm{Pl}}\right)$ and Higgs $v e v \simeq 246 \mathrm{GeV}$, which can produce strong bounds of $m_{\phi}$ and $\Lambda_{\phi}$. It is phenomenologically important that the one-loop allowed upper bound of the radion mass is about five times larger than the tree-level one, but the radion is still lighter than the Kaluza-Klein modes.
\end{abstract}

*E-mail: dawn@muon.kaist.ac.kr

†E-mail: hslee@muon.kaist.ac.kr 


\section{INTRODUCTION}

Recently it was known that the large difference between the Planck scale $\Lambda_{\mathrm{Pl}} \sim 10^{18}$ $\mathrm{GeV}$ and the electroweak scale $\Lambda_{\mathrm{EW}} \sim 10^{2} \mathrm{GeV}$ (so-called the hierarchy problem) can be produced by introducing the compact extra dimension(s). There are two models with extra dimension(s). One is the ADD model (large extra-dimension model) [1], where the extra dimensions are below $O(1) m m\left(\gg 1 / M_{W}\right)$ and the extra submanifold is at least two dimensional. Although the ADD model resolves the hierarchy problem, it causes another hierarchy problem between the electroweak scale and the radius of the extra dimensions. This comes from the power law behavior of the hierarchy, that is, $\Lambda_{\mathrm{EW}}=\left(R \Lambda_{\mathrm{Pl}}\right)^{-n /(2+n)} \Lambda_{\mathrm{Pl}}$, where $R$ and $n$ are the typical radius and the dimension of the extra submanifold, respectively. The other is the Randall-Sundrum (RS) model (small extra-dimension model) [2], where the classical RS metric is $d s_{\mathrm{RS}}^{2}=e^{-2 k r_{c}|y|} \eta_{\mu \nu} d x^{\mu} d x^{\nu}-r_{c}^{2} d y^{2}$ and the extra manifold is a one-dimensional orbifold $S^{1} / Z_{2} . \quad k$ is smaller than the 5-dimensional Planck mass $M_{(5)} \sim$ $M_{\mathrm{Pl}} \simeq 2 \times 10^{18} \mathrm{GeV}, \pi r_{c}$ is the interbrane distance, and the hidden and visible branes are at $y=0$ and $y=\pi$, respectively. The electroweak scale can be derived from the Planck scale via $\Lambda_{\mathrm{EW}}=e^{-k r_{c} \pi} \Lambda_{\mathrm{Pl}}$, where $e^{-k r_{c} \pi}$ is the geometrical warp factor appearing in the metric of our brane. The unwanted extra hierarchy problem does not exist due to the exponential dependence of the hierarchy on the radius $r_{c}$ unlike the ADD theory.

When a kind of modulus stabilization with a bulk scalar is added to the RS theory (since it is impossible to determine the value of the interbrane distance $\sim O\left(10 / M_{(5)}\right)$ from the dynamics of the "original" RS model) and a kind of dimensional reduction is done, the modulus field (radion) couples to the Standard Model (SM) fields like $\bar{\phi} T_{\mu}^{\mu} / \Lambda_{\phi}$ [3, 何, where $\bar{\phi}$ is the physical field of the radion, $\Lambda_{\phi}$ is a dimensionful coupling constant of order $\Lambda_{\mathrm{EW}}$, and $T_{\mu}^{\mu}$ is the trace of the energy-momentum tensor of the SM fields. The magnitudes of $\Lambda_{\phi}$ and the radion mass $m_{\phi}$ are phenomenologically important, since they are related to decay rates and cross sections. There are two kinds of considerations on these parameters until now. One is experimental, and the other is theoretical. The experimental consideration is related to the experimentally measured quantities, for example, the decay rates and production cross sections of radion, and the radion loop corrections to the weak mixing angle [5-9]. It is noteworthy that the branching ratio of the radion into two gluons is the largest for $m_{\phi} \lesssim 160 \mathrm{GeV}$, although it comes from the trace anomaly at the one-loop level. The recent L3 results on the SM Higgs search excluded $\Lambda_{\phi} \lesssim 600 \mathrm{GeV}$ for $m_{\phi} \lesssim 100 \mathrm{GeV}$ [7]. The constraint from the weak mixing angle with errors of order $O(0.1) \%$ can exclude $\Lambda_{\phi} \sim 500$ $\mathrm{GeV}$ for $m_{\phi} \sim 500 \mathrm{GeV}$ [8]. And the lower bound of the radion mass is about $O(1) \mathrm{GeV}$ from limits of neutrino phenomenology and TASSO and CLEO on inclusive B decays [9]. The theoretical one is on the perturbative unitarity [7, 10. The perturbative unitarity for $h h \rightarrow h h$ ( $h:$ SM Higgs boson) is broken for relatively small $\Lambda_{\phi} \lesssim 200 \mathrm{GeV}$ for $m_{\phi} \sim 600$ $\mathrm{GeV}$ [7], and the unitarity bound in the $W_{L}^{+} W_{L}^{-} \rightarrow h \phi$ process is $m_{\phi} \lesssim 2500 \mathrm{GeV}$ at $\Lambda_{\phi}=1$ $\mathrm{TeV}[10]$.

In this paper, we have constrained the two parameters up to one-loop level in terms of two conditions, warp factor $e^{-k r_{c} \pi}=O\left(\Lambda_{\mathrm{EW}} / \Lambda_{\mathrm{Pl}}\right)$ and Higgs vev $\simeq 246 \mathrm{GeV}$ (vev= vacuum expectation value). The constraint, warp factor $=O\left(\Lambda_{\mathrm{EW}} / \Lambda_{\mathrm{Pl}}\right)$, was imposed to solve the hierarchy problem [2]. From the above conditions, the one-loop allowed bounds

of $m_{\phi}$ and $\Lambda_{\phi}$ are $0.8 \mathrm{GeV} \lesssim m_{\phi} \lesssim 260 \mathrm{GeV}$ and $1400 \mathrm{GeV} \lesssim \Lambda_{\phi} \lesssim 1500 \mathrm{GeV}$, which 
can be stronger than the previous constraints except the lower bound of radion mass. It is noteworthy that the one-loop upper bound of the radion mass $m_{\phi}$ is about five times larger than the tree-level one of about $49 \mathrm{GeV}$, and still smaller than the masses of the low-lying Kaluza-Klein (KK) modes.

This paper is organized as follows: In Section II, we discuss what fields give important contributions to the effective potential, and analyze the contributions of the scalar and KK mode sectors and those of the fermion and gauge sectors. In Section III, we find the bounds on $m_{\phi}$ and $\Lambda_{\phi}$ from warp factor $=O\left(\Lambda_{\mathrm{EW}} / \Lambda_{\mathrm{Pl}}\right)$ and Higgs $v e v \simeq 246 \mathrm{GeV}$. The conclusions are in Section IV.

\section{ONE-LOOP EFFECTIVE POTENTIAL}

When the hierarchy problem is solved with only the one-loop corrections of bulk fields, they generate a very light radion [11], which is excluded by experiments of neutrino phenomenology and $B$ decays [9]. Therefore, the stabilization mechanism at the tree level is required [11, 12]. The mechanism proposed by Goldberger and Wise [13] can be a promising one, because they stabilized the modulus without any severe fine-tuning of the parameters in the full theory. In the Goldberger-Wise stabilization mechanism, there is a bulk scalar field $\Phi\left(x^{\mu}, y\right)$ which has large quartic self-interactions on the hidden and visible branes alone, and a $y$-dependent vev $\tilde{\Phi}(y)$. After replacing the field $\Phi\left(x^{\mu}, y\right)$ in the original Lagrangian with its vev $\tilde{\Phi}(y)$ and integrating the Lagrangian over $y$, we have the modulus stabilizing potential. The typical energy of this mode is the expectation value of an operator $p_{y}=-\frac{1}{r_{c}} \frac{\partial}{\partial y}$ to $\tilde{\Phi}(y)$ of order $O\left(10^{-2}\right) k$, which makes the effective gravitational coupling constant $\left(\right.$ Energy $\left./ M_{(5)}\right)$ of the bulk scalar about $O\left(10^{-2}\right)$ by the 5 -dimensional Planck suppression. Thus the quantum correction of the 5-dimensional graviton to the bulk scalar vev $\tilde{\Phi}(y)$ is unimportant in the full theory. And the quantum corrections from the brane self-interactions of $\Phi$ can shift the vevs of the bulk scalar at the branes, $v_{v}$ and $v_{h}$, where $v_{v}=\tilde{\Phi}(\pi)$ and $v_{h}=\tilde{\Phi}(0)$. These corrections can change only the coefficients of the radion potential and be equivalent to rescaling of the coefficients.

The 4 -dimensional graviton coupling to the radion is $\mathcal{L}_{\text {grav }}=-\left(2 M_{(5)}^{3} / k\right) e^{-2 \phi / \Lambda_{\phi}} \mathcal{R}_{(4)}$ [2]:3], where $\mathcal{R}_{(4)}$ is the 4-dimensional Ricci scalar. When the radion $\phi$ is replaced with $\phi=\phi_{0}+\bar{\phi}$, where $\phi_{0}$ is the vev of $\phi$, the one-graviton coupling constant to the physical field of the radion $\bar{\phi}$ is Planck-suppressed, $O\left(\Lambda_{\mathrm{EW}} / M_{\mathrm{Pl}}\right) \ll 1$, since $\mathcal{R}_{(4)}$ has linear terms of the graviton field $h_{\mu \nu}(x)=\left(g_{\mu \nu}(x)-\eta_{\mu \nu}\right) / \sqrt{8 \pi G_{N}}$ in the weak field approximation. So we can neglect the quantum effects of the 4-dimensional graviton. There are low-lying KK modes of the bulk scalar and the bulk graviton with masses of $O(1) \mathrm{TeV}$ and nonrenormalizable couplings suppressed by TeV scale [14]. The effects of these low-lying KK modes on the electroweak scale physics are absorbed into the renormalizations of the Planck and $\mathrm{TeV}$ brane tension terms $V_{\text {Planck }}+V_{\mathrm{TeV}} e^{-4 \phi / \Lambda_{\phi}}$ [12]. Since the radion has highly nonrenormalizable self-interactions such as $V_{\phi}$ in Eq. (3), we treated the radion self-interactions only at the tree level. For example, the non-renormalizable quantum gravity has a serious problem that the one-loop effective action on the mass-shell is dependent on the gauge fixing parameters [15, breaking the DeWitt-Kallosh theorem [16]. Therefore, we consider the loop corrections of the KK modes of bulk fields and the SM particles to the radion effective potential in our brane. 


\section{A. Scalar and KK mode sectors}

To make its kinetic term canonically normalized, we rescale the Higgs field $H(x) \rightarrow$ $e^{k b(x) / 2} H(x)$ [4], where $k b(x) / 2=\phi(x) / \Lambda_{\phi}$. After this rescaling, the scalar sector has the Lagrangian density [3, [1]

$$
\begin{aligned}
\mathcal{L}_{\text {scalar }}= & D_{\mu} H^{\dagger} D^{\mu} H+\frac{1}{2}(\partial \phi)^{2}-V_{H}(H, \phi)-V_{\phi}(\phi) \\
& +\frac{\partial_{\mu} \phi}{\Lambda_{\phi}}\left(H^{\dagger} D^{\mu} H+\text { h.c. }\right)+\left(\frac{\partial \phi}{\Lambda_{\phi}}\right)^{2} H^{\dagger} H .
\end{aligned}
$$

The potentials $V_{H}$ 표 and $V_{\phi}$ [13] are

$$
V_{H}(H, \phi)=\lambda\left(H^{\dagger} H-\frac{1}{2} v_{0}^{2} e^{-2 \phi / \Lambda_{\phi}}\right)^{2},
$$

and

$$
\begin{aligned}
V_{\phi}(\phi)= & 4 k e^{-4 \phi / \Lambda_{\phi}}\left(v_{v}-v_{h} e^{-\epsilon \phi / \Lambda_{\phi}}\right)^{2}\left(1+\frac{\epsilon}{4}\right) \\
& -k \epsilon v_{h} e^{-(4+\epsilon) \phi / \Lambda_{\phi}}\left(2 v_{v}-v_{h} e^{-\epsilon \phi / \Lambda_{\phi}}\right),
\end{aligned}
$$

where $\epsilon \simeq m^{2} / 4 k^{2}$ is about $1 / 40(k / m$ is of order unity) to solve the hierarchy problem [13. The ranges of $v_{0}, v_{v}^{2 / 3}, v_{h}^{2 / 3}, k$, and the bulk scalar mass $m$ are of order $O(0.1) M_{(5)}$ in order to avoid a large hierarchy in the RS theory and to maintain the classical RS metric or, equivalently, neglect the loop corrections of the 5-dimensional quantum gravity. In the Landau gauge, three massless would-be Goldstone bosons appeared in the calculation unlike the unitary gauge. The fifth term in Eq. (1) is the mixing term of radion and Higgs fields with two derivatives. It has to be considered in the canonical normalizations of the kinetic terms of the radion and Higgs fields [4]. The effective potential from the scalar sector in the $\overline{\mathrm{MS}}$ scheme is

$$
\begin{aligned}
V_{\text {eff }}^{\text {scalar }}(h, \phi)= & V_{\text {tree }}(h, \phi)+V_{1 \text { loop }}^{\text {scalar }}(h, \phi) \\
= & V_{\phi}(\phi)+\frac{\lambda}{4}\left(h^{2}-v_{0}^{2} e^{-2 \phi / \Lambda_{\phi}}\right)^{2} \\
& +\frac{1}{4(4 \pi)^{2}}\left\{\lambda^{2}\left(3 h^{2}-v_{0}^{2} e^{-2 \phi / \Lambda_{\phi}}\right)^{2}\left(\log \left[\lambda\left(3 h^{2}-v_{0}^{2} e^{-2 \phi / \Lambda_{\phi}}\right) / \mu^{2}\right]-\frac{3}{2}\right)\right. \\
& \left.\quad+3 \lambda^{2}\left(h^{2}-v_{0}^{2} e^{-2 \phi / \Lambda_{\phi}}\right)^{2}\left(\log \left[\lambda\left(h^{2}-v_{0}^{2} e^{-2 \phi / \Lambda_{\phi}}\right) / \mu^{2}\right]-\frac{3}{2}\right)\right\},
\end{aligned}
$$

where $\mu$ is a renormalization scale. The term with a coefficient $\lambda^{2}$ in Eq. (四) came from the physical Higgs field, and the 3 Goldstone bosons $G^{0}, G^{+}$and $G^{-}$gave the next term with a coefficient $3 \lambda^{2}$. The KK modes originated from the bulk scalar can couple to the Higgs field with trilinear couplings [17] which give the effective quartic term of the Higgs field $\frac{c_{\mathrm{KK}}}{4} h^{4}$ at the tree level. The contributions of the low-lying KK modes to $c_{\mathrm{KK}}$ are much smaller than the SM one-loop corrections in Eq. (4), and thus can be neglected.

But the one-loop corrections from the KK modes of the bulk scalar and graviton can contribute considerably to the radion potential as $V_{1 \text { loop }}^{\mathrm{KK}}(\phi)=\delta V_{\mathrm{Planck}}^{\mathrm{KK}}+\delta V_{\mathrm{TeV}}^{\mathrm{KK}} e^{-4 \phi / \Lambda_{\phi}}$ [11, 12, 
where $\delta V_{\text {Planck }}^{\mathrm{KK}}$ and $\delta V_{\mathrm{TeV}}^{\mathrm{KK}}$ are shifts from the classical Planck and TeV brane tensions $V_{\text {Planck }}=$ $-V_{\mathrm{TeV}}=24 M_{(5)}^{3} k>0$ [2] respectively, belonging to the tension shifts $\delta V_{\text {Planck }}$ and $\delta V_{\mathrm{TeV}}$ of $V_{\Lambda}\left(r_{c}\right)=\delta V_{\text {Planck }}+\delta V_{\mathrm{TeV}} e^{-4 k r_{c} \pi}$ in Ref. [13]. For small $\delta V_{\mathrm{TeV}}$, the addition of $V_{\Lambda}$ to the potential $V_{\phi}$ in Eq. (3) gives a minimum for large $k r_{c}$, and the adjustment of the tension shift $\delta V_{\text {Planck }}$ on the Planck brane can make the effective four-dimensional cosmological constant vanish 13, 11]. Since the shift of the TeV brane tension $\delta V_{\mathrm{TeV}}^{\mathrm{KK}}$ is absorbed into the renormalization of the $\mathrm{TeV}$ brane tension term [12] $S_{\mathrm{tension}}^{\mathrm{TeV}}=-\int d^{4} x \sqrt{-g_{\mathrm{TeV}}} V_{\mathrm{TeV}}$ [2, 13], it can not be obtained from calculation and can only be determined by a renormalization condition relating it to observable quantities [11]. Because the 4-dimensional cosmological constant of order $10^{-120} M_{\mathrm{Pl}}^{4} \ll \Lambda_{\mathrm{EW}}^{4}\|18\|$ is regarded as the minimum value of the effective potential $V_{\text {eff }}(h, \phi)$ of the RS theory, the value of the potential can be approximated as zero at the observed values of $h$ and $\phi$, which should be a stable minimum point of the potential [11. The two renormalization conditions are expressed as

$$
V_{\text {eff }}=0 \quad \text { and } \quad \frac{\partial V_{\text {eff }}}{\partial h}=\frac{\partial V_{\text {eff }}}{\partial \phi}=0
$$

at the observed values of $h$ and $\phi$. From these first and second conditions, we can determine the shifts $\delta V_{\text {Planck }}$ and $\delta V_{\mathrm{TeV}}^{\mathrm{KK}}$ respectively, if all the other parameters are known. The size of the shift $\delta V_{\mathrm{TeV}}^{\mathrm{KK}}$ can be of order $O\left(\Lambda_{\mathrm{Pl}}^{4} / 100\right)$ much smaller than $\left|V_{\mathrm{TeV}}\right|$. It is noteworthy that the sign of the KK mode contribution is important in the vacuum stability since it can change the shape of potential. If $\delta V_{\mathrm{TeV}}^{\mathrm{KK}}$ is fixed to be negative and $\left|\delta V_{\mathrm{TeV}}^{\mathrm{KK}}\right|$ increases, the potential becomes deeper and has more parameter points which give stable vacua. But when the sign is reversed, the number of the allowed points gets smaller, and thus the negative $\delta V_{\mathrm{TeV}}^{\mathrm{KK}}$ can be favored in terms of stability.

\section{B. Fermion and Vector sectors}

Since we used the $x^{\mu}$-dependent rescaling $\psi(x) \rightarrow e^{3 k b(x) / 2} \psi(x)$ for a fermion $\psi$, the radion-fermion interaction Lagrangian is $\mathcal{L}_{\phi \psi \bar{\psi}}=i\left(3 / 2 \Lambda_{\phi}\right) \bar{\psi} \gamma^{\mu} \psi \partial_{\mu} \phi$ [4]. The Lagrangians for the gauge bosons are similar to that of the Higgs boson. The background field method [19] and the tadpole method [20] showed easily that the fermions and gauge bosons of the Standard Model do not give any one-loop contributions to the radion potential although the interaction Lagrangians are non-renormalizable. Therefore, only the Higgs potential received the loop corrections from these particles. The contributions of the fermion and vector sectors to the effective potential are

$$
\begin{aligned}
V_{\mathrm{eff}}^{\text {fer }+ \text { vec }}(h) & =V_{1 \text { loop }}^{\text {fermion }}(h)+V_{1 \operatorname{loop}}^{\text {vector }}(h) \\
& =\frac{1}{(4 \pi)^{2}}\left\{-3 T^{2}\left(\log \frac{T}{\mu^{2}}-\frac{3}{2}\right)+\frac{3}{2} W^{2}\left(\log \frac{W}{\mu^{2}}-\frac{5}{6}\right)+\frac{3}{4} Z^{2}\left(\log \frac{Z}{\mu^{2}}-\frac{5}{6}\right)\right\},
\end{aligned}
$$

where $T=\frac{1}{2} Y_{t}^{2} h^{2}, W=\frac{1}{4} g^{2} h^{2}$, and $Z=\frac{1}{4}\left(g^{2}+g^{\prime 2}\right) h^{2}$ [21]. The $g$ and $g^{\prime}$ are the gauge coupling constants of $\mathrm{SU}(2)_{L} \times \mathrm{U}(1)_{Y}$, and the $Y_{t}$ is the top quark Yukawa coupling constant. Since the fermion contribution is proportional to (Yukawa coupling constant) ${ }^{4}$, only the top quark contribution is considered. The difference of constants in the parentheses of the fermion sector and the gauge sector came from the gauge dependent term $(1-\xi) k^{\mu} k^{\nu} /\left(k^{2}-\right.$ 
$\left.\xi m^{2}\right)(\xi=0)$ of the gauge boson propagator. This can be seen qualitatively and easily in the tadpole method [20]. Therefore, adding the contribution of the KK modes of the bulk fields, the final effective potential up to the one-loop level is

$$
\begin{aligned}
V_{\text {eff }}(h, \phi)= & V_{\text {tree }}(h, \phi)+V_{1 \text { loop }}^{\text {scalar }}(h, \phi)+V_{1 \text { loop }}^{\text {fermion }}(h)+V_{1 \text { loop }}^{\text {vector }}(h)+V_{1 \text { loop }}^{\mathrm{KK}}(\phi) \\
= & V_{\phi}(\phi)+\frac{\lambda}{4}\left(h^{2}-v_{0}^{2} e^{-2 \phi / \Lambda_{\phi}}\right)^{2} \\
& +\frac{1}{4(4 \pi)^{2}}\left\{\lambda^{2}\left(3 h^{2}-v_{0}^{2} e^{-2 \phi / \Lambda_{\phi}}\right)^{2}\left(\log \left[\lambda\left(3 h^{2}-v_{0}^{2} e^{-2 \phi / \Lambda_{\phi}}\right) / \mu^{2}\right]-\frac{3}{2}\right)\right\} \\
& \left.+3 \lambda^{2}\left(h^{2}-v_{0}^{2} e^{-2 \phi / \Lambda_{\phi}}\right)^{2}\left(\log \left[\lambda\left(h^{2}-v_{0}^{2} e^{-2 \phi / \Lambda_{\phi}}\right) / \mu^{2}\right]-\frac{3}{2}\right)\right\} \\
& +\frac{1}{(4 \pi)^{2}}\left\{-3 T^{2}\left(\log \frac{T}{\mu^{2}}-\frac{3}{2}\right)+\frac{3}{2} W^{2}\left(\log \frac{W}{\mu^{2}}-\frac{5}{6}\right)+\frac{3}{4} Z^{2}\left(\log \frac{Z}{\mu^{2}}-\frac{5}{6}\right)\right\} \\
& +\delta V_{\mathrm{Planck}}^{\mathrm{KK}}+\delta V_{\mathrm{TeV}}^{\mathrm{KK}} e^{-4 \phi / \Lambda_{\phi}} .
\end{aligned}
$$

Note that all the one-loop corrections are of the same order $O\left(\Lambda_{\mathrm{EW}}^{4} / 100\right)$ for our parameter range. The vevs $v$ and $\phi_{0}$ of the Higgs neutral component field $h(x)$ and the radion field $\phi(x)$ are determined by the stationary condition of the effective potential, when the fields $h$ and $\phi$ are assumed to be independent of spacetime coordinates. It is reasonable that we should consider the region of $v$ and $\phi_{0}$ where the validity of perturbation and the condition of real $V_{\text {eff }}$ are satisfied.

\section{BOUNDS ON $M_{\phi}$ AND $\Lambda_{\phi}$}

In the Randall-Sundrum theory, the extra dimension must have a finite size about $O\left(10 / M_{\mathrm{Pl}}\right)$ to solve the hierarchy problem [2,13], which gives the constraint, warp factor $e^{-k r_{c} \pi}=O\left(\Lambda_{\mathrm{EW}} / \Lambda_{\mathrm{Pl}}\right)$. And in the Standard Model, the vev of the Higgs field is about 246 $\mathrm{GeV}$. This can give a constraint on the Higgs vev of the effective potential in the RS model. But when the quantum corrections of radion are added to the SM particles, the experimentally determined vev of the Higgs field may be much different from the SM value, that is, the corrections may destroy the above constraint. When we consider the quantum corrections of the radion to the masses of the SM particles using the Lagrangian $\mathcal{L}_{\phi \psi \bar{\psi}}=i\left(3 / 2 \Lambda_{\phi}\right) \bar{\psi} \gamma^{\mu} \psi \partial_{\mu} \phi$, the pole masses of leptons and heavy quarks, for example, are

$$
\begin{aligned}
m_{f}^{\text {pole }} \simeq & m_{f}^{\mathrm{SM}}\left\{1+\frac{1}{(4 \pi)^{2}}\left(\frac{m_{f}}{\Lambda_{\phi}}\right)^{2} \int_{0}^{1} d x\left[\frac{9}{4}(3 x-1) \frac{\Delta\left(m_{f}^{\text {pole }}\right)}{m_{f}^{2}}\right.\right. \\
& \left.\left.+\left(1+x-\frac{9}{4}(1+x)(1-x)^{2}\left(\frac{m_{f}^{\text {pole }}}{m_{f}}\right)^{2}-\frac{9}{2}(3 x-1) \frac{\Delta\left(m_{f}^{\text {pole }}\right)}{m_{f}^{2}}\right) \ln \frac{\Delta\left(m_{f}^{\text {pole }}\right)}{\mu^{2}}\right]\right\},
\end{aligned}
$$

where $m_{f}^{\mathrm{SM}}$ are the renormalized masses with the SM loop corrections alone, $m_{f}$ are the mass parameters in the renormalized Lagrangian, $\mu$ is a renormalization scale, and $\Delta\left(m_{f}^{\text {pole }}\right)=$ $-x(1-x)\left(m_{f}^{\text {pole }}\right)^{2}+x m_{\phi}^{2}+(1-x) m_{f}^{2}$. If $\Lambda_{\phi} \lesssim O\left(m_{f} / 4 \pi\right)$, then the radion correction can be large so that the shift of the Higgs vev from the SM one can be large. (This argument is 
similarly applied to the gauge bosons and the Higgs boson.) So the condition $v \simeq 246 \mathrm{GeV}$ can be spoilt for $\Lambda_{\phi} \lesssim O\left(m_{f} / 4 \pi\right)$. But this small range is excluded by the constraint from the weak mixing angle that $\Lambda_{\phi} \lesssim 300 \mathrm{GeV}$ are excluded for $m_{\phi} \leq 1000 \mathrm{GeV}[\mathbb{8}$. Therefore, it can be natural that the physical pole mass $\left(e . g . m_{f}^{\text {pole }}\right)$ is almost equal to the SM mass (e.g. $\left.m_{f}^{\mathrm{SM}}\right)$. This means that the Higgs $v e v \simeq 246 \mathrm{GeV}$ can be a physical constraint on our effective potential for the natural range of $\Lambda_{\phi}$. The radion corrections are typically smaller than $O(0.1) \%$ of $m_{f}^{\mathrm{SM}}$, so we can expect that the errors of the Higgs vev from the radion contributions are smaller than $O(0.1) \%$.

The procedure to determine $m_{\phi}$ and $\Lambda_{\phi}$ is as follows. First, we find a parameter point of $\left(v_{0}, v_{v}, v_{h}, m, \delta V_{\mathrm{TeV}}^{\mathrm{KK}}\right)$ which gives a stable vacuum satisfying the two constraints, warp factor $e^{-k r_{c} \pi}=O\left(\Lambda_{\mathrm{EW}} / \Lambda_{\mathrm{Pl}}\right)$ and Higgs vev $v \simeq 246 \mathrm{GeV}$. Note that $\delta V_{\mathrm{TeV}}^{\mathrm{KK}}$ is not considered for the tree-level potential. Of course, the point has to be in the region where perturbation is valid and $V_{\text {eff }}$ is real. Next, the values of $m_{\phi}$ and $\Lambda_{\phi}$ are determined from that point by means of equations $m_{\phi}^{2}=\frac{\partial^{2} V_{\phi}}{\partial \phi^{2}}\left(\phi_{0}\right)$ and $\Lambda_{\phi}=\sqrt{6} M_{\mathrm{Pl}} e^{-k b_{0} / 2}$. Since when a minimum point is found, $\delta V_{\text {Planck }}^{\mathrm{KK}}$ can be determined trivially via the first equation in Eq. (55), we do not include the parameter $\delta V_{\text {Planck }}^{\mathrm{KK}}$. Because the RS metric $d s_{\mathrm{RS}}^{2}$ is a solution of the "classical" 5-dimensional Einstein's equation, in order to maintain the classical metric we choose parameter ranges where the loop effects of the quantum gravity can be neglected.

For the tree level potential, the allowed region for the 5-dimensional Planck mass of a typical size $M_{(5)}=0.8 M_{\mathrm{Pl}}$ and the Higgs mass $m_{h}=125 \mathrm{GeV}$ is $0.4 \mathrm{GeV} \lesssim m_{\phi} \lesssim 49 \mathrm{GeV}$ and $760 \mathrm{GeV} \lesssim \Lambda_{\phi} \lesssim 5900 \mathrm{GeV}$ from Fig. 1, where $\Lambda_{\phi}=\sqrt{6} M_{\mathrm{Pl}} e^{-k b_{0} / 2}$ and $m_{\phi}^{2}=\frac{\partial^{2} V_{\phi}}{\partial \phi^{2}}\left(\phi_{0}\right)$. Because of the exponential dependence of $\Lambda_{\phi}=\sqrt{6} M_{\mathrm{P}} e^{-k b_{0} / 2}$, the large change of $\Lambda_{\phi} \sim$ $O(100) \%$ corresponds to the very small change of $k b_{0} / 2 \sim O(1) \%$, whose central value is about $36\left(k r_{c} \simeq 12\right)$. This means that the range of the radion vev $\phi_{0}$ is very narrow. From Fig. Q, most of the allowed parameter points (about 93 percent) are concentrated around the line $\Lambda_{\phi}=1070 \mathrm{GeV}$, and thus the point with a smaller or larger $\Lambda_{\phi}$ is rare. Therefore, the naturally allowed region can be much narrower in $\Lambda_{\phi}$ than the above allowed one.

For the one-loop effective potential, the allowed region for the 5-dimensional Planck mass $M_{(5)}=0.8 M_{\mathrm{Pl}}$ and the Higgs mass $m_{h}=125 \mathrm{GeV}$ is $0.8 \mathrm{GeV} \lesssim m_{\phi} \lesssim 260 \mathrm{GeV}$ and $1400 \mathrm{GeV} \lesssim \Lambda_{\phi} \lesssim 1500 \mathrm{GeV}$ from Fig. 3 (the central value of $k b_{0} / 2$ is still about 36 ). From Fig. 2, almost all the data are focused at $\Lambda_{\phi}=1490 \mathrm{GeV}$ like the tree level case. Therefore, we can conclude that the naturally allowed regions are similar for the tree and one-loop cases, and there is a small shift of the central value of $\Lambda_{\phi}$ (or equivalently $\phi_{0}$ ) due to the one-loop corrections. When $\delta V_{\mathrm{TeV}}^{\mathrm{KK}}$ and other parameters are changed continuously, the allowed region in Fig. 3 can be broader in $\Lambda_{\phi}$. From numerical analysis, we have found that only the negative values of $\delta V_{\mathrm{TeV}}^{\mathrm{KK}}$ can be allowed. As discussed in Section II, negative values of the tension shift $\delta V_{\mathrm{TeV}}^{\mathrm{KK}}$ produce many parameter points which were not allowed at the tree level, and considerable parts of these new points make the radion mass sufficiently larger than the tree-level upper bound of the mass. It is phenomenologically noteworthy that the one-loop upper bound of the radion mass $m_{\phi}$ is rather larger than the tree-level one by about five times. But the radion is still the first signal of the RS theory lighter than the lowest-lying KK mode with a mass of order $O(1) k e^{-k b_{0} / 2} \simeq 0.8 \Lambda_{\phi}$ [14,22], because the radion mass is smaller than about $260 \mathrm{GeV}$. And the branching ratios of the radion into gluon or $W$ boson pairs are dominant according to the mass $m_{\phi}$ [5.][]. 
The tree level perturbative unitarity can not be stronger than the two constraints, warp factor $e^{-k r_{c} \pi}=O\left(\Lambda_{\mathrm{EW}} / \Lambda_{\mathrm{Pl}}\right)$ and Higgs $v e v v \simeq 246 \mathrm{GeV}$, in constraining $m_{\phi}$ and $\Lambda_{\phi}$, since the unitarity bound in the $h h \rightarrow h h$ process is $\Lambda_{\phi} \gtrsim 300 \mathrm{GeV}$ at $m_{\phi} \lesssim 1000 \mathrm{GeV}$ [7] and the bound in the $W_{L}^{+} W_{L}^{-} \rightarrow h \phi$ process is $m_{\phi} \lesssim 2500 \mathrm{GeV}$ at $\Lambda_{\phi}=1 \mathrm{TeV}$ [10]. For the radion mass $m_{\phi} \lesssim 100 \mathrm{GeV}$, the bounds from the SM Higgs search [7] are helpful, since $\Lambda_{\phi} \lesssim 600 \mathrm{GeV}$ can be excluded. The limits from neutrino phenomenology and TASSO and CLEO on inclusive $B$ decays give lower bounds of the radion mass about $1 \mathrm{GeV}$ [9], which agree to our lower bound. And the region $\Lambda_{\phi} \sim 750 \mathrm{GeV}$ for cutoff $\Lambda=1 \mathrm{TeV}$ is excluded by the constraint from the weak mixing angle [8], since $e^{-k r_{c} \pi}=O\left(\Lambda_{\mathrm{EW}} / \Lambda_{\mathrm{Pl}}\right)$ and $v \simeq 246$ $\mathrm{GeV}$ make $m_{\phi}$ less than about $260 \mathrm{GeV}$. Most of the previous constraints gives only lower bounds. Only the unitary bound in the $W_{L}^{+} W_{L}^{-} \rightarrow h \phi$ process gives the weak upper bound of $m_{\phi}$. Compared with the above constraints, the $e^{-k r_{c} \pi}=O\left(\Lambda_{\mathrm{EW}} / \Lambda_{\mathrm{Pl}}\right)$ and $v \simeq 246 \mathrm{GeV}$ constraints can produce the bounds of $m_{\phi}$ and $\Lambda_{\phi}$ strongly, except the mass lower bound.

\section{CONCLUSIONS}

Assuming the Goldberger-Wise mechanism in the Randall-Sundrum theory, we considered the allowed regions of $m_{\phi}$ and $\Lambda_{\phi}$ from two constraints, warp factor $e^{-k r_{c} \pi}=$ $O\left(\Lambda_{\mathrm{EW}} / \Lambda_{\mathrm{Pl}}\right)$ and Higgs $v e v v \simeq 246 \mathrm{GeV}$, by means of the tree level and one-loop level potentials. The allowed regions are $0.4 \mathrm{GeV} \lesssim m_{\phi} \lesssim 49 \mathrm{GeV}$ and $760 \mathrm{GeV} \lesssim \Lambda_{\phi} \lesssim 5900 \mathrm{GeV}$ for the tree level potential, and $0.8 \mathrm{GeV} \lesssim m_{\phi} \lesssim 260 \mathrm{GeV}$ and $1400 \mathrm{GeV} \lesssim \Lambda_{\phi} \lesssim 1500 \mathrm{GeV}$ for the one-loop level potential. It is phenomenologically important that the one-loop allowed upper bound of radion mass $m_{\phi}$ is considerably larger than the tree-level one, but the radion is still the first experimental signature of the $\mathrm{RS}$ model, since it is lighter than the KK modes. The mass of the radion is less than about $260 \mathrm{GeV}$, and thus it decays into gluon or $W$ boson pairs dominantly. And its loop contributions are small due to the average value of the effective coupling constant $v / \Lambda_{\phi} \sim 1 / 6$ (the average effective fine structure constant is about $\left.2 \times 10^{-3} \lesssim \alpha_{\mathrm{em}}\right)$. The conditions of $e^{-k r_{c} \pi}=O\left(\Lambda_{\mathrm{EW}} / \Lambda_{\mathrm{Pl}}\right)$ and $v \simeq 246 \mathrm{GeV}$ can give the strong bounds of $m_{\phi}$ and $\Lambda_{\phi}$.

Note Added: After completing this paper, we received an interesting paper by U. Mahanta [23], who considered the one-loop contributions of the radion and the KK modes of graviton to the Higgs potential. We did not include the radion contribution due to the non-renormalizability in Section II. In Ref. [23], the one-loop contributions via some approximations are too small to cause any instability of the classical vacuum in the valid region of perturbation theory. Therefore, these contributions do not change our results significantly.

\section{ACKNOWLEDGEMENT}

We would like to thank Prof. Pyungwon Ko for discussions. This work is supported by the Brain Korea 21 Project and grant No. 1999-2-111-002-5 from the interdisciplinary research program of the KOSEF. 


\section{REFERENCES}

[1] N. Arkani-Hamed, S. Dimopoulos and G. Dvali, Phys. Lett. 429B, 263 (1998); I. Antoniadis, N. Arkani-Hamed, S. Dimopoulos and G. Dvali, Phys. Lett. 436B, 257 (1998).

[2] L. Randall and R. Sundrum, Phys. Rev. Lett. 83, 3370 (1999).

[3] W.D. Goldberger and M.B. Wise, Phys. Lett. 475B, 275 (2000).

[4] C. Csáki, M. Graesser, L. Randall, and J. Terning, Phys. Rev. D62, 045015 (2000).

[5] G.F. Giudice, R. Rattazzi and J.D. Wells, Nucl. Phys. B595, 250 (2001).

[6] U. Mahanta and A. Datta, Phys. Lett. 483B, 196 (2000).

[7] S. Bae, P. Ko, H. S. Lee, and J. Lee, Phys. Lett. 487B, 299 (2000).

[8] J.E. Kim, B. Kyae and J.D. Park, hep-ph/0007008.

[9] U. Mahanta and S. Mohanty, Phys. Rev. D62, 083003 (2000).

[10] U. Mahanta, hep-ph/0004128.

[11] J. Garriga, O. Pujolàs and T. Tanaka, hep-th/0004109.

[12] W.D. Goldberger and I.Z. Rothstein, Phys. Lett. 491B, 339 (2000).

[13] W. D. Goldberger and M. B. Wise, Phys. Rev. Lett. 83, 4922 (1999).

[14] W. D. Goldberger and M. B. Wise, Phys. Rev. D60, 107505 (1999).

[15] S. Ichinose, Phys. Lett. 284B, 234 (1992); Nucl. Phys. B395, 433 (1993).

[16] B.S. DeWitt, Phys. Rev. 162, 1195 (1967); R. Kallosh, Nucl. Phys. B78, 293 (1974).

[17] C. Csáki, M.L. Graesser and G.D. Kribs, hep-th/0008151.

[18] A.G. Riess et al., Astron. J. 116, 1009 (1998); P.M. Garnavich et al., Astrophys. J. 509, 74 (1998).

[19] M.E. Peskin and D.V. Schroeder, "An Introduction to Quantum Field Theory" (Addison-Wesley Publishing Company, 1995).

[20] M. Sher, Phys. Rep. 179, 273 (1989); S.Y. Lee and A.M. Sciaccaluga, Nucl. Phys. B96, 435 (1975).

[21] C. Ford, D.R.T. Jones, P.W. Stephenson, and M.B. Einhorn, Nucl. Phys. B395, 17 (1993).

[22] T. Gherghetta and A. Pomarol, Nucl. Phys. B586, 141 (2000); H. Davoudiasl, J.L. Hewett and T.G. Rizzo, Phys. Rev. Lett. 84, 2080 (2000).

[23] U. Mahanta, hep-ph/0011148. 


\section{FIGURES}

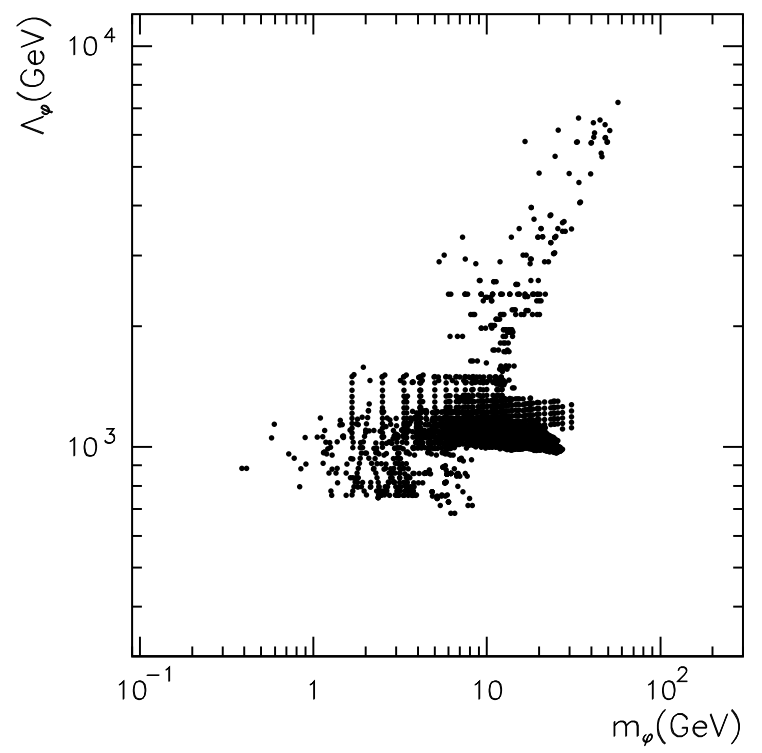

FIG. 1. The allowed parameter points in $m_{\phi}$ and $\Lambda_{\phi}$ space for the tree level potential $\left(M_{(5)}=0.8 M_{\mathrm{Pl}}\right.$ and $\left.m_{h}=125 \mathrm{GeV}\right)$.

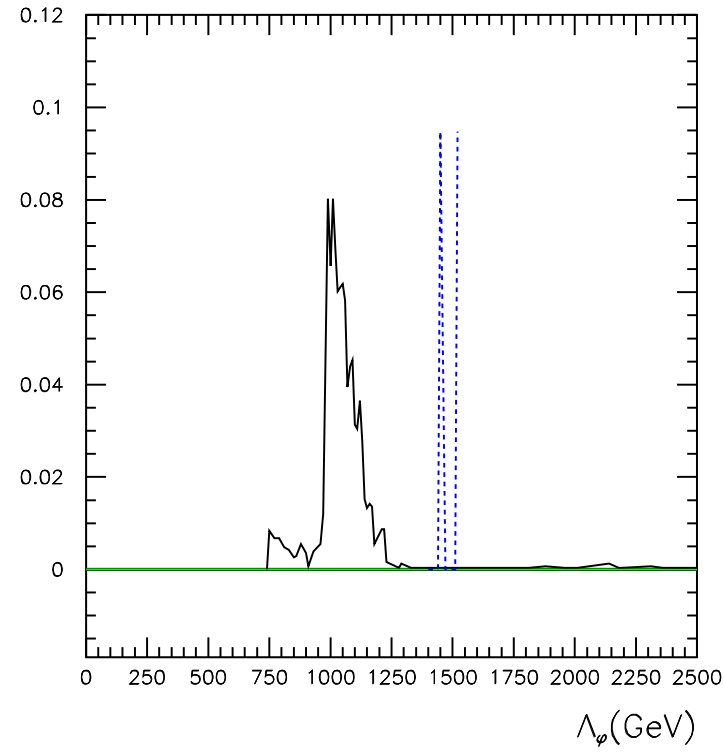

FIG. 2. The $y$ coordinates of the graphs are proportional to the numbers of allowed parameter points. The solid and dotted lines denote the allowed points for the tree and one-loop level potentials, respectively. The tree-level points with $\Lambda_{\phi}>2500 \mathrm{GeV}$ are not shown. 


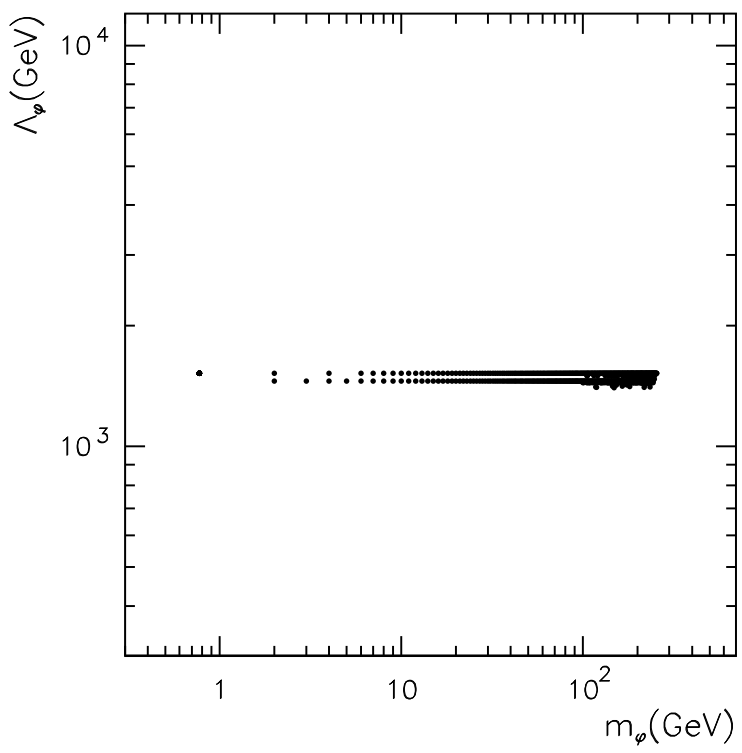

FIG. 3. The allowed points for the one-loop effective potential $\left(M_{(5)}=0.8 M_{\mathrm{Pl}}\right.$ and $m_{h}=125$ $\mathrm{GeV}$ ). 